Cahiers $d u$ MONDE RUSSE

\section{Cahiers du monde russe}

Russie - Empire russe - Union soviétique et États indépendants

$49 / 4 \mid 2008$

Destins individuels et terreur. Jeunesse dans la société post-stalinienne

\title{
Dmitrij Chmelnizki, Die Architektur Stalins
}

\section{Gábor T. Rittersporn}

\section{(2) OpenEdition}

\section{Journals}

Édition électronique

URL : https://journals.openedition.org/monderusse/6960

DOI : $10.4000 /$ monderusse. 6960

ISSN : $1777-5388$

Éditeur

Éditions de l'EHESS

Édition imprimée

Date de publication : 28 décembre 2008

Pagination : 771-774

ISBN : 978-2-7132-2197-2

ISSN : $1252-6576$

Référence électronique

Gábor T. Rittersporn, «Dmitrij Chmelnizki, Die Architektur Stalins », Cahiers du monde russe [En ligne], 49/4 | 2008, mis en ligne le 24 décembre 2009, consulté le 03 septembre 2022. URL : http://

journals.openedition.org/monderusse/6960; DOI : https://doi.org/10.4000/monderusse.6960

Ce document a été généré automatiquement le 3 septembre 2022

Tous droits réservés 


\title{
Dmitrij Chmelnizki, Die Architektur Stalins
}

\author{
Gábor T. Rittersporn
}

\section{RÉFÉRENCE}

Dmitrij CHMELNIZKI, Die Architektur Stalins. Studien zu Ideologie und Stil. Stuttgart : Ibidem, 2007, 2 vol., $475+208$ p. (Soviet and Post-Soviet Politics and Society).

1 Pour l'honnête homme, l'architecture de l'ère stalinienne se résume à peu de chose : il connaît les bâtiments somptueux qui ont commencé à occuper l'espace urbain à partir du milieu des années 1930 et surtout ceux qui ont été construits dans les années 1950 ; il a une idée des imposants gratte-ciel qui ont surgi à Moscou après-guerre, il sait que le sous-sol de la ville abrite le métro le plus fastueux du monde; il a peut-être entendu parler du projet jamais réalisé d'un gigantesque bâtiment dans la capitale de l'URSS...

2 L'ouvrage de Dmitrij Chmelnizki comble bien quelques lacunes, mais son auteur est trop soucieux de ne pas s'écarter des sentiers battus pour apporter des éléments vraiment nouveaux à nos connaissances. Car, de fait, serait-il excessif d'attendre d'une histoire de l'architecture soviétique qu'elle inclue un chapitre sur l'habitat rural et poursuive ainsi le travail pionnier de Basile Kerblay ${ }^{1}$ ? Est-il déraisonnable d'espérer que l'on prête un jour attention à l'urbanisme singulier des quartiers ouvriers faits de baraquements, de cabanes et d'abris à moitié enfouis sous terre ? Oserait-on se demander si les maisons de bois qu'on n'a cessé de bâtir et de décorer jusque dans les années 1950, même à Moscou, ne pourraient documenter les goûts d'une époque et cet art du possible qu'est, après tout, l'architecture? Serait-il extravagant de s'intéresser à l'aménagement et au réaménagement astucieuxdes anciens immeubles pour loger des institutions de toute espèce et des gens dans des appartements communautaires?

3 Le livre de Chmelnizki est loin de satisfaire toutes ces curiosités. Rien sur l'architecture industrielle ni sur celle du projet chéri de l'État-parti, les parcs de la culture, pourtant étudiés par Katharina Kucher'2. C'est que Chmelnizki se focalise sur ce qu'il considère, à 
tort ou à raison, comme l'architecture de Stalin. Certes, il documente deux ou trois interventions du Leader dans ce domaine. Mais les sources indiquent que celui-ci se plaisait surtout à critiquer les plans de reconstruction de Moscou, si bien que nous ne savons pas grand-chose de ses prédilections dans le domaine de l'architecture en tant que telle. Stalin s'était mêlé du concours du pharaonique Palais des Soviets: au vu du résultat, il n'aimait pas plus l'avant-garde que le classicisme. Mais malgré tout, il aimait passer une partie de son temps dans une datcha marquée par l'héritage constructiviste: Chmelnizki publie une des rares photos du pavillon, plutôt raté, de Sotchi.

Que dire donc de l'architecture du dictateur? L'auteur a certes le mérite d'avoir épluché les débats des années 1930, 1940 et 1950 sur le style idéal - débats qui traitent d'une multitude de bâtiments de l'époque; il se fonde également sur une quantité impressionnante de textes parus dans les périodiques professionnels; il en va de même de divers concours abondamment commentés dans les revues spécialisées. Chmelnizki en a extrait des discours suffisamment disparates pour qu'on puisse parler d'un consensus sur un langage architectural unique et sur les efforts des autorités pour l'imposer.

Or, à y regarder de près, les composantes de l'architecture de la période stalinienne sont très hétérogènes. Les canons du classicisme antique et des XVIII $-\mathrm{XIX}^{\mathrm{e}}$ siècles y cohabitent avec un large éventail d'éléments qui vont de la Renaissance italienne jusqu'au constructivisme, tantôt affiché, tantôt habilement mélangé à d'autres ingrédients. Éclectisme décourageant pour qui veut tout ramener à un dénominateur commun. L'architecte, dans un même projet, pouvait affubler une façade empruntée aux palais florentins d'une colonnade vaguement corinthienne, et couronner le tout d'une décoration digne des arcs de triomphe russes $\mathrm{du} \mathrm{XIX}^{\mathrm{e}}$ siècle.

6 La seule chose certaine que l'on peut dire de ce cocktail de styles est qu'il est fort conservateur par rapport à l'architecture expérimentale des années 1920. C'est à peu près tout ce que l'auteur réussit à exprimer. Pour le reste, tout au long de l'ouvrage, il fait le deuil de l'avant-garde. Mais, ce faisant, il oublie qu'il n'y a eu aucun pays avant-guerre où l'architecture moderne soit parvenue à s'affirmer dans le paysage urbain. Elle a eu la préférence des commanditaires de bâtiments privés de taille plutôt modeste, comme des villas, et a surtout été utilisée pour l'habitat bon marché. Mais la plupart du temps, l'avant-garde est restée aux marges.

7 En France, par exemple, on peut trouver des analogies entre les logements sociaux de Paris dans les années 1930et les quartiers fonctionnalistes de Francfort ou de Hambourg dans les années 1920. Par contre, la construction haut de gamme des années 1930 s'est rarement dispensée d'éléments fin de siècle ou même de l'époque haussmannienne. Des bâtiments publics de grand prestige comme le palais de Chaillot faisaient fi des préceptes de l'avant-garde. Entre les deux guerres mondiales, celle-ci ne pouvait commander autant le respect que les constructions traditionnelles. Le centre de Washington offre la preuve qu'il est réducteur de comparer uniquement au paradigme nazi le style soviétique qui émergeait dans les années 1930. Construits entre 1935 et 1943, la Cour suprême, les Archives nationales, la Galerie nationale d'art et le Mémorial de Jefferson témoignent autant d'un goût conservateur et d'un éclectisme déroutant que ce que l'on peut observer en URSS. Du reste, on ne peut sans doute pas prétendre que le Rockefeller Center, qui a tant fasciné les architectes soviétiques, doive plus à l'esprit de l'avant-garde qu'à celui de l'art nouveau.

8 Pendant longtemps, on n'a pas découvert de moyens radicalement neufs pour manifester la distinction et la grandeur d'établissements prestigieux, d'espaces vénérables et de 
régimes un tant soit peu mégalomanes. Certains États avaient la chance d'avoir installé depuis belle lurette leurs institutions dans des demeures imposantes d'aristocrates, de rois et d'empereurs d'un passé plus ou moins lointain ou dans des palais dont les auteurs s'inspiraient de modèles datant d'avant le $\mathrm{xx}^{\mathrm{e}}$ siècle. D'autres étaient moins favorisés : la Finlande fut ainsi l'un des pays de l'architecture moderne. Mais ceci n'empêcha pas le gouvernement d'élever au début des années 1930 un parlement d'un style aussi conservateur et indéfinissable que celui du malheureux Palais des Soviets ou (pourquoi pas ?) de l'ambassade soviétique qui sera construite après la guerre.

Chmelnizki voit bien le lien entre la nécessité d'ériger des bâtiments publics et l'abandon des recettes de l'avant-garde, mais il se perd trop dans les méandres des discussions sur le style du prolétariat triomphant pour se rendre compte que le contexte international contribue à favoriser le tournant conservateur qu'il situe vers 1932. C'est le moment où les représentants de l'architecture moderne sont écartés du concours pour le Palais des Soviets. Le Corbusier a beau protester auprès de Lunačarskij et envoyer avec quelques collègues un long télégramme à Stalin, rien n'y fait. Peu après, son projet pour le Musée d'art moderne de Paris sera rejeté au profit d'un autre, assez éloigné de son architecture du futur et qui rappelle la façade de la Bibliothèque Lénine.

Sona S. Hoisington a montré que l'élaboration du style choisi pour le Palais des Soviets avait été plus complexe qu'on ne le croit $^{3}$. Le modèle définitif était à peu près indéfinissable, mêlant plusieurs styles et se caractérisant essentiellement par son côté monumental. S'appuyant entre autres sur des documents provenant des archives municipales de Moscou, Hoisington a suivi les avatars de la construction, envisagée, dans un premier temps, près du Kremlin en recourant à la participation des tenants étrangers de l'avant-garde. L'emplacement de la cathédrale du Christ-Sauveur ne fut choisi que plus tard. Il impliquait davantage la démesure que la modération de l'avant-garde, ce qui ne motivait pas pour autant un retour aux formes traditionnelles.

11 On peut se demander si ces formes se sont développées aussi simplement que Chmelnizki le croit. Les matériaux publiés ne sont peut-être que la partie visible de l'iceberg. Les fonds de l'Union des architectes de l'URSS et de celle de Moscou au RGALI (Rossijskij gosudarstvennyj Arhiv literatury i iskusstva - Archives d'État de Russie pour la littérature et les arts), ainsi que les documents de l'administration protéiforme de l'Architecture de Moscou, conservés aux archives de la ville, risquent de modifier les idées reçues, surtout en ce qui concerne les préférences des commanditaires. Il serait également utile de fouiller dans les archives d'Ukraine, de Biélorussie et même de Géorgie, où les autorités étaient friandes de bâtiments prestigieux. L'hypothèse d'une interaction souple entre les goûts manifestement incertains des commanditaires et le choix des architectes est tout aussi concevable que le diktat du Leader et de ses sbires.

$12 \mathrm{Au}$ fond, les débats des contemporains sur l'architecture stalinienne ne définissaient aucun programme, se focalisant sur ce que les architectes étaient censés ne pas faire. Le constructivisme et le fonctionnalisme n'étaient pas moins vertement critiqués que ce que l'on prenait pour un attachement excessif à la tradition ou même pour l'éclectisme qui régnait pourtant en maître. Le formalisme, dénoncé à cor et à cri, était aussi difficile à saisir en architecture qu'en littérature, dans le théâtre, les arts plastiques ou le cinéma.

13 Il est tout à l'honneur de Chmelnizki de ne pas interrompre son récit à la mort du dictateur. Il poursuit jusqu'au début des années 1960 où un vaste programme de construction de logements et un concours pour un nouveau Palais des Soviets dans l'enceinte du Kremlin remettent à l'ordre du jour le style des années 1920. Vite rebaptisé 
Palais des congrès, le projet victorieux n'aura rien à voir avec le phantasme mégalomane des années 1930. Un certain nombre de participants au concours présentèrent néanmoins des projets s'inspirant du style de l'énorme bâtisse, d'autres ne répudièrent pas entièrement l'héritage du passé récent.L'auteur ne mentionne pas que, dès le milieu des années 1950, le jury d'une autre compétition opta pour un pavillon, de toute évidence inspiré par le modernisme de l'après-guerre, qui allait représenter l'URSS à l'Exposition universelle de Bruxelles. De manière erronée, Chmelnizki n'attribue le tournant qu'à la condamnation des coûts énormes de l'architecture somptueuse de l'ère stalinienne. Il n'entrevoit pas que cette nouvelle simplicité était inséparable d'un projet de société qui voulait rompre avec ce que les Soviétiques entendaient à l'époque par stalinisme et revaloriser l'esprit des années 1920. Le projet est resté sans lendemain. En revanche, les éléments stylistiques de l'époque post-révolutionnaire s'étaient banalisés. Il serait sans doute instructif de se demander pourquoi le régime autoritaire de l'URSS n'éprouva aucune difficulté à accepter les avatars de ce style dans les années 1960 et plus tard encore.

Tout compte fait, il s'agit d'un livre assez décevant. Il est trop axé sur le destin de l'avant-garde, sur la recherche d'un style stalinien unique et sur des discussions somme toute stériles. On peut en extraire des informations sur les dits, voire les non-dits, d'architectes et de théoriciens plus ou moins connus. Mais il y aura sans doute des lecteurs pour s'intéresser avant tout au second volume de l'ouvrage, qui comporte 435 illustrations. Puisées pour la plupart dans les publications des années 1930 à 1950, celles-ci représentent des bâtiments aussi bien que des projets alternatifs. Chmelnizki a fourni un gros travail pour réunir cette documentation et ne peut être tenu pour responsable de la qualité lamentable des images. Celle-ci incombe à l'éditeur, qui vend pourtant son livre à un prix excessif sur lequel il est fort à parier qu'il ne reverse rien à l'auteur.

\section{NOTES}

1. Basile Kerblay, L'isba d'hier et d'aujourd'hui (Lausanne : L'Âge d'homme 1973).

2. Katharina Kucher, Der Gorki-Park. Freizeitkultur im Stalinismus, Cologne : Böhlau, 2007, p. 69-97, 187-211.

3. Sona S. Holsington, "Ever Higher": The Evolution of the Project for the Palace of Soviets ", Slavic Review, 62 (1) 2003, p. 41-68. 